\title{
Squeezed Protection in Elastic Optical Networks Subject to Multiple Link Failures
}

\author{
K. D. R. Assis, R. C. Almeida Jr, L. P. Dias and H. Waldman
}

\begin{abstract}
Elastic optical network (EON) is fast becoming a key solution for designing optical network with better usage of spectrum resources or other objectives of interest to tenant and/or operators. A primary concern of EONs is to protect the network against failures of its elements, because this kind of event can provoke the loss of substantial amount of traffic. In this paper, we propose a new mixed integer linear programming (MILP) formulation for protecting the network traffic against multiple link failures. The key idea is to use bandwidth squeezing together with grooming capability to provide few extra traffic for protection and guarantee a minimum bandwidth for each source-destination node pair under multiple failure events. The proposed formulation solves the virtual topology design problem jointly with the grooming, routing, modulation and spectrum allocation (RMSA) tasks. Due to the non-deterministic polynomial time (NP-hard) nature of the proposed MILP formulation, a heuristic strategy (referred to as two-step MILP) for large networks is also proposed. The solutions and performance of the proposed MILP formulation and two-step MILP analyzed through case studies in a small network. In addition, the performance of three large networks is assessed for cases scenarios where connections are under different service-level agreement (SLA). In view of proposed formulation and two-step MILP, it is possible to identify the configurations that ensure better usage of spectrum resources with different kinds of protection against single or multiple link failures.
\end{abstract}

Index Terms-Elastic Optical Networks, Optimization, Routing, Survivability, Modulation Format, Virtualization

\section{INTRODUCTION}

$\mathbf{E}$ LASTIC optical networks (EONs) were introduced to address the limitation of spectrum usage in existing wavelength-division multiplexing (WDM)-based optical networks by providing spectrum-efficient and scalable transport of $100 \mathrm{Gbit} / \mathrm{s}$ services and beyond, [1-3]. It also aggregates the throughput per fiber link to approximately $10-100 \mathrm{~Tb} / \mathrm{s}$. However, adverse events over EON networks, such as optical fiber cuts, can disrupt communications for millions of users, which can lead to a great loss of data and revenue. Other components may also incur even more harmful failures, but they are not as frequent [4-7], so this paper will consider only the effects of fiber cuts.

Most researches have focused on improving the service availability of these networks against multiple failures, often assuming that every user demands a bandwidth equal

Karcius Day R. Assis and Leonardo P. Dias are with the Electrical and Computer Engineering Department, Federal University of Bahia (UFBA), Salvador-BA, Brazil, e-mail: karcius.assis@ufba.br

Raul C. Almeida Jr is with Department of Electronics and Systems, Universidade Federal de Pernambuco (UFPE), Recife-PE, Brazil.

Helio Waldman is with the Department of Communications Engineering, State University of Campinas (Unicamp), Campinas-SP, Brazil.

Manuscript received XXXX YY, ZZZZ; revised YYYYY XX, ZZZZ. to the full wavelength capacity. However, with EONs, the fixed wavelength channels, which are defined in traditional WDM networks, no longer exist; instead, bandwidth-variable wavebands share the entire spectrum in one fiber in a "gridless" manner. Indeed, EON proposes various grids that are different from the $50 \mathrm{GHz}$ fixed grid of traditional WDM systems. The minimum slot size defined by the ITU-T G.709 is $12.5 \mathrm{GHz}$.

Regarding routing strategies in high layer or virtual topology, traffic grooming allows the aggregation of traffic at intermediate nodes from different source-destination node pairs [8, 9]. Such grooming techniques allow the establishment of optical paths carrying several traffic flows in a contiguous block of spectrum without inserting guard bands in between, therefore minimizing the spectrum usage. The newly introduced aspects of EONs with grooming allow proposals for new forms of protection capability against failures. To meet this requirement, dedicated path protection (DPP) has appeared as the most common survivability option, however it incurs a $100 \%$ resource overhead that remains unused most of the time [10]. Thus, fairly often, DPP is a source of overprovisioning.

To minimize the resource footprint of DPP, a technique referred to as squeezing protection or bandwidth squeezing restoration (BSR) was leveraged that allows the network operator to tune the amount of available bandwidth under failure conditions [2, 11-14]. BSR allows the squeezing of working lightpath capacity from the original to a reduced capacity as the "best-effort" recovery. Considering the fact that network resources are always limited, the BSR technique has the desirable feature of enhancing network connectivity and availability by partially recovering the bandwidth of an affected optical channel, while complying with the guarantees agreed in the SLA [15].

This paper addresses the conflicting needs of the network to be parsimonious in the assignment of the network spectral resources while still meeting the requirements of users from a multi-tenant environment, which may be heterogeneous. If the requirements are not homogeneous, as illustrated on Fig. 1 for just two source-destination pairs, offering the same protection for all pairs may result in a needless waste of bandwidth. In order to tackle this issue, the paper starts by reviewing the basic modes of protection at the path level, defines some parametric metrics for protection overhead and vulnerability to fiber cuts, and derives some feasibility conditions for these metrics under single and multiple failures. Then, proceeding to the network level, it starts with the presentation of a mixed integer linear programming (MILP) formulation that derives an optimal virtual topology over a small physical substrate that achieves minimal commitment of the spectral resources while meeting the agreed protection targets for all source-destination 


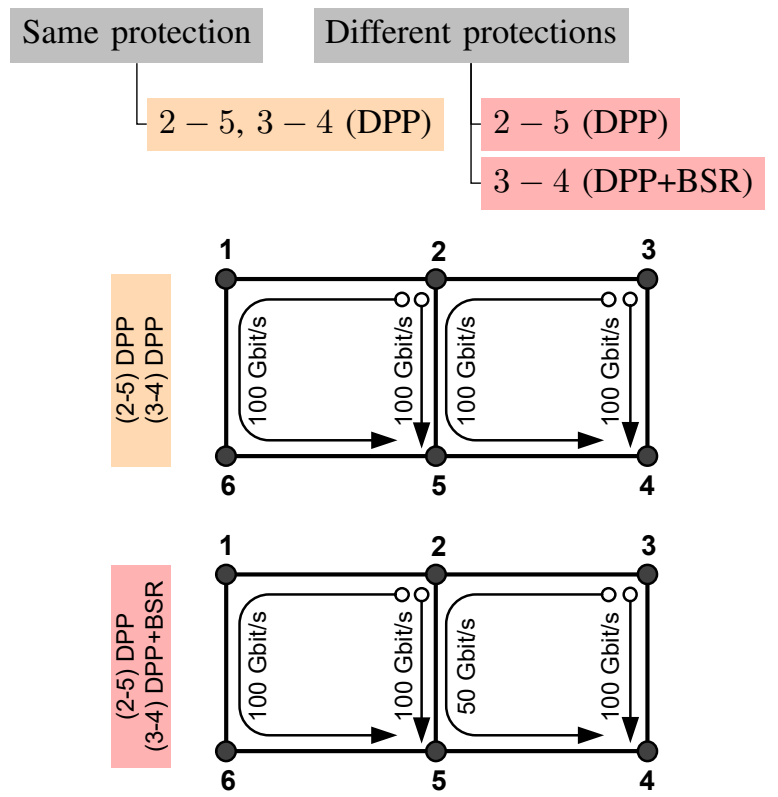

Fig. 1: Kinds of protection planning for two lightpaths (2-5 and 3-4) over a physical substrate.

pairs. Since this formulation would be impractical for large networks, it is then somewhat tweaked in order to obtain a twostep MILP that generates non-optimal, but still good, solutions for the same purpose. Finally, the proposed two-step MILP is applied to a few real networks and the results are presented and briefly discussed.

The remainder of the paper is organized as follows: Section II is a brief review of related work published since 2009 . Section III begins by laying out the theoretical dimensions of the research and is concerned with the methodology used for this study. In Section IV the MILP is presented. In Section $\mathrm{V}$ the heuristic for large instances is proposed. Section VI presents the findings of the research, focusing on a small and large networks. Finally, Section VII gives a brief summary, a critique of the findings and identifies areas for further research.

\section{RELATED WORK}

Bandwidth Spectrum Restoration (BSR) was first proposed in [2] in 2009 as a timely functionality to be provided by the proposed SLICE network architecture, which came to underpin the emergence of elastic optical networks. The new architecture is enabled by emerging technologies that are now mature enough to provide the elasticity capability needed to squeeze the bandwidth whenever needed. Its basic aim is to overcome the waste of spectral resources due to their overprovision generated by the rigid offer of bandwidth by the fixed grid of legacy WDM networks, given the heterogeneous demand for bandwidth from the users. In order to provide a better match between offer and demand, a flexible grid is proposed that is now standardized. BSR is then necessary to provide parsimony in the commitment of spectral resources to the spare capacity.

In [16], the authors draw attention to a distinctive category of reductions in the extra bandwidth required for protection capability in EONs. The central idea is that the strict differentiation between working and protection paths no longer applies, so that a total bandwidth may be reserved through the use of as many disjoint paths as needed (obviously constrained to the connectivity of the physical topology or other aspect of interest). Therefore, the approach must coordinate the distribution of the traffic and reserved bandwidth among the routes with some optimization aim, for instance spectrum saving. In a failure event that affects one of the adopted disjoint paths, the paper shows the existence of a trade-off between the protection overhead to be paid during regular operation and the guarantee of a minimum fraction of the original traffic by the SLA during the repair time of a single failure on one of the participating disjoint paths.

In 2016, a comprehensive survey of the state-of-the-art of survivable EON's was presented [15], showing several refinements of the SLICE concept since its first inception. Several technical solutions are discussed other than BSR, such as the hitless defragmentation of the protection bandwidth, sharing of protection and transponder resources, spectrum splitting and conversion, etc., thus describing a complex landscape of the EON survivability issue, in which BSR stands as a unique feature.

A growing body of literature has focused on BSR restoration/protection in EONs [13, 14, 17]. In [13], the authors discuss a combination of bitrate squeezing with multipath restoration in a SDN-controlled architecture supported by SBVT's, using an extended version of the OpenFlow protocol enabled to operate on EON's. In [14], the authors propose a MILP formulation that jointly solves the virtual topology design and physical topology design problems, with connections complying with customized service-level agreement (SLA) requirements. In [17], The authors present a quantitative assessment of the resilience gains of EON's when compared with legacy WDM, not only for classic one-to-one traffic, but also for cloud- and content-oriented demands that tend to dominate the traffic in present-day communication networks.

Despite the proven benefits of grooming, previous studies have not treated in much detail the combination of routing (single or multiple paths) and protection with grooming and RSA. In particular, with grooming, it may not be feasible to set up virtual links with protection between all node pairs due to a limited number of resources such as optical transmitters, receivers or regenerators [18]. Therefore, there still remain important challenges regarding grooming and virtualization in optical networks with protection, mainly with the 5th generation of mobile networking (5G) on our doorstep, and [19] provides evidence to support our claim for a detailed study in that area. The above supports the need to study protection with routing and grooming to design EONs in a scalable and cost-effective manner.

\section{Survivability Design in Optical Networks with TRAFFIC SQUEEZING}

In this section, the principle behind the most commonly used protection schemes is reviewed and discussed. Firstly, DPP and BSR are discussed in Subsection A. Then, in Subsection B, a new approach known as Partitioning Dedicated Path Protection (PDPP) is presented. Afterwards, in Subsection C, in order 
to clarify the advantages/disadvantages and requirements of implementing each of the above mentioned protection mechanisms, a detailed mathematical description of the problem under single link failure is provided, as discussed in [14]. Lastly, in Subsection D, a multiple link failure formulation is proposed and an extensive discussion is provided.

\section{A. Dedicated Path Protection and Bandwidth Squeezing Restoration}

A large and growing body of literature addresses the problem of BSR restoration/protection in EONs [12,14, 17]. For instance, Fig. 2a shows a 6-node network with an active virtual link between nodes 2 and 5 mapped on the physical link connecting the same source-destination pair. Let us suppose that the virtual link is transporting $100 \mathrm{Gbit} / \mathrm{s}$ of traffic. An alternative to protect this traffic against a failure of its working physical path is by the activation of another physical path with the same $100 \mathrm{Gbit} / \mathrm{s}$ of capacity on a disjoint route, as shown in Fig $2 \mathrm{~b}$

When a link failure occurs, for example in link 2-5, the disrupted virtual link is obviously restored using the backup path 2-1-6-5. This approach is known as DPP and is frequently dealt with in the literature on survivability [10]. Spectrum saving investigation is a major concern in elastic networking field [1]. On the other hand, an inflexible protection scheme such as DPP was envisaged without major objections in the old framework of the fixed grid, where it was seen as just another source of overprovisioning among many others. However, whereas this kind of protection has now become a major source of waste on spectrum usage, current accounts have so far failed to resolve the contradiction between dedicated protection and the need to save spectrum. What is not yet clear is the impact of chosen protection scheme on planning network costs or user satisfaction. Since spectrum is treated as a scarce resource, a spectrally more efficient substitute for DPP is certainly needed.

Preliminary works on BSR were undertaken by [2, 11] as an alternative to DPP. By drawing on the concept of BSR, these studies have argued that, on a failure event, the total traffic between two nodes may be reduced in a suitable way complying with the pre-accorded SLA. This approach is treated in this paper as DPP with bandwidth squeezing restoration (DPP+BSR) and is shown in Fig. 2c One can note that, in a failure event on the working traffic, the original $100 \mathrm{Gbit} / \mathrm{s}$ may be squeezed to $50 \mathrm{Gbit} / \mathrm{s}$ (which means a reduction of $50 \%$ ). Therefore, only an additional $50 \mathrm{Gbit} / \mathrm{s}$ has to be reserved for protection purposes and the total network bitrate would be $150 \mathrm{Gbit} / \mathrm{s}$ during normal operation (value much lower than the required $200 \mathrm{Gbit} / \mathrm{s}$ in DPP).

\section{B. $P D P P$}

For a better understanding, an example of PDPP and PDPP+BSR is shown, respectively, in Fig. 3a and Fig. 3b. In the first case, notice that if the requested $100 \mathrm{Gbit} / \mathrm{s}$ of traffic is equally partitioned among two distinct lightpaths and an extra lightpath with $50 \mathrm{Gbit} / \mathrm{s}$ is provisioned, the same $150 \mathrm{Gbit} / \mathrm{s}$ of traffic as in DPP+BSR (Fig. 2c) is enough to guarantee that any single link failure provides a maximum traffic reduction of

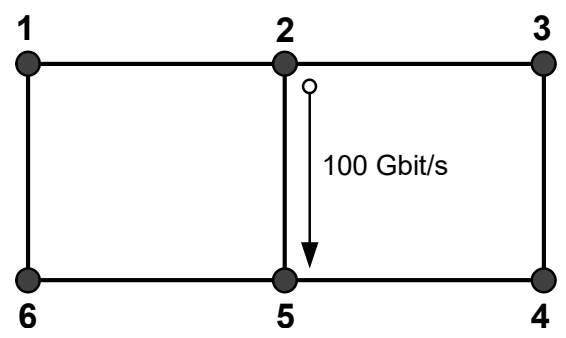

(a)

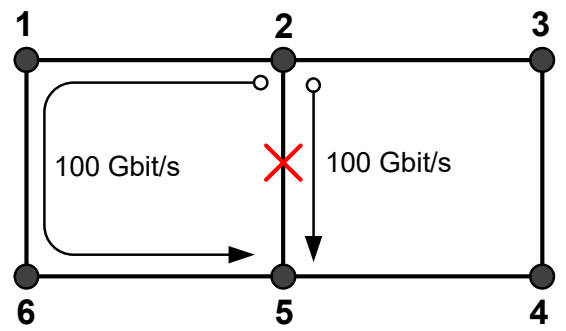

(b)

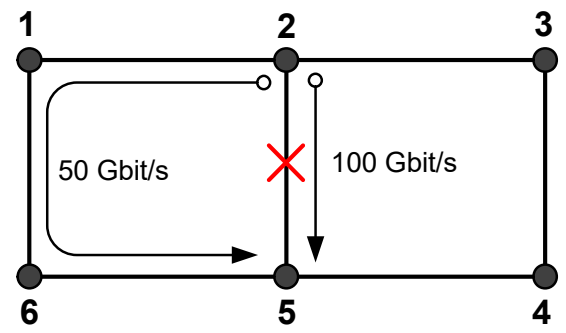

(c)

Fig. 2: Examples of network protection approaches presented in Section II-A: (a) No protection; (b) DPP; (c) DPP+BSR, [14].

$50 \mathrm{Gbit} / \mathrm{s}$. Therefore, differently from the DPP+BSR presented in Fig. 2c, which experience a traffic reduction (i.e. squeezing) of $50 \mathrm{Gbit} / \mathrm{s}$ under link failure, PDPP is able to maintain the required $100 \mathrm{Gbit} / \mathrm{s}$. On the other hand, if squeezing is added to PDPP (Fig. 3b), traffic can be partitioned among three lightpaths and just $120 \mathrm{Gbit} / \mathrm{s}$ is required to transport the requested $100 \mathrm{Gbit} / \mathrm{s}$ and maintain $80 \mathrm{Gbit} / \mathrm{s}$ (20\% traffic squeezing) under link failure. Therefore, a lower amount of total bandwidth as well as a higher value of residual traffic under link failure is achieved when PDPP+BSR is compared to $\mathrm{DPP}+\mathrm{BSR}$. PDPP+BSR is also able to reduce the total bit rate assigned to PDPP with the cost of experiencing traffic squeezing.

The saving of spectral resources afforded by the PDPP partitioning technique has a price, in the form of more management complexity and additional transponders when compared with classical (e.g. DPP) schemes.

\section{Single Path Failure}

Let $\Lambda^{s d}$ be the committed traffic demand from source node $s$ to destination node $d$ under regular operation. Then, in order to provide some degree of protection to this traffic, the network must keep some amount $\alpha(s, d) \Lambda^{s d}$ of additional spectral resources reserved to this aim, where $\alpha(s, d)$ is a measure of the protection overhead paid by the network to protect service to node pair $(s, d)$ while a failing critical component is 


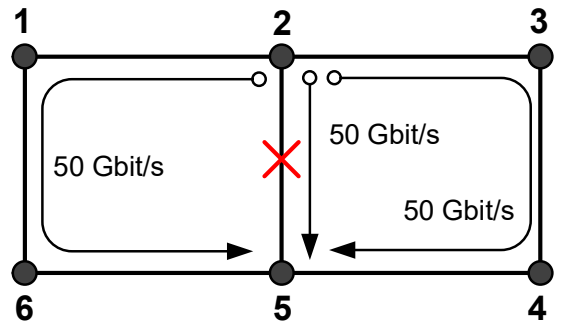

(a)

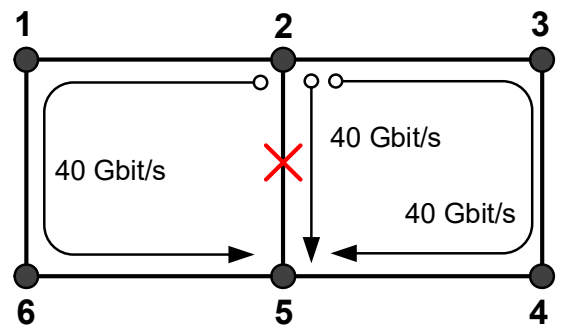

(b)

Fig. 3: Examples of network protection approaches presented in Section II-B: (a) PDPP with $\alpha(s, d)=0.5$; (b) PDPP+BSR with $\alpha(s, d)=0.2$ and $\beta(s, d)=0.2$, [14].

repaired or replaced. In traditional DPP, since no tolerance for bandwidth squeezing is accepted and only two disjoint paths are adopted, $\alpha(s, d)=1$. In contrast, in the PDPP approach, $0 \leq \alpha(s, d) \leq 1$ may be assigned, which means that the total bandwidth reserved for working and protection on connection $s-d$ can be set as $[1+\alpha(s, d)] \Lambda^{s d} \leq 2 \Lambda^{s d}$, which can be lower than that of DPP protection. In order to provide service to as many users as possible with its limited spectral resources, the network would like $\alpha(s, d)$ to be as little as possible. Ideally from the network perspective, $\alpha(s, d)$ should then be zero.

In order to set $\alpha(s, d)=0$ while still complying with the SLA, the contracting user must agree with some traffic squeezing during the repair time. We then assume that, during this time, the SLA allows the committed traffic to be reduced to $[1-\beta(s, d)] \Lambda^{s d}$, where $\beta(s, d)$ is a squeezing factor. Of course, from the perspective of the user, $\beta(s, d)$ should also be ideally zero, so that service is not degraded at all by the failure. However, it is shown in this sub-Section that setting $\alpha(s, d)=\beta(s, d)=0$ is not feasible, even though it is feasible to set either $\alpha(s, d)$ or $\beta(s, d)$ to zero, but never both at the same time.

Let the overall working-plus-protection bandwidth $[1+\alpha(s, d)] \Lambda^{s d}$ assigned to the $(s, d)$ pair be carried over $g(s, d)$ disjoint paths. In order to minimize the worst-case loss caused by a single failure among the $g(s, d)$ disjoint paths, the traffic is uniformly distributed among them. Then, upon the onset of a single failure, the surviving bandwidth will be given by $[1+\alpha(s, d)][1-1 / g(s, d)] \Lambda^{s d}$, which must be larger than or equal to the guaranteed bandwidth $[1-\beta(s, d)] \Lambda^{s d}$, resulting in the following inequality:

$$
g(s, d)[\alpha(s, d)+\beta(s, d)] \geq[1+\alpha(s, d)],
$$

which proves that $\alpha(s, d)$ and $\beta(s, d)$ cannot be both set to zero at the same time. Handling (1) to isolate $\alpha(s, d)$ and $\beta(s, d)$ on each side of the inequality yields the following feasibility condition for compliance with the SLA under single failures:

$$
\alpha(s, d) \geq[1-\beta(s, d) g(s, d)] /[g(s, d)-1] \text {. }
$$

with equality holding for a best compromise between a minimal $\alpha(s, d)$ given $\beta(s, d)$, and/or a minimal $\beta(s, d)$ given $\alpha(s, d)$. Notice that if $\alpha(s, d)$ is set to zero, then $\beta(s, d)$ is at least $1 / g(s, d)$, leading optimally to $\beta(s, d)=1$ for $g(s, d)=$ 1 , with no protection at all; $\beta(s, d)=1 / 2$ for $g(s, d)=2$, i.e. $50 \%$ squeezing for two disjoint paths; $33.3 \%$ squeezing for 3 disjoint paths; $25 \%$ for 4 disjoint paths, etc.. On the other hand, setting $\beta(s, d)=0$ means that the protection overhead $\alpha(s, d)$ must be at least $100 \%$ for $g(s, d)=2$, as in DPP; $50 \%$ for $g(s, d)=3 ; 33.3 \%$ for $g(s, d)=4$; and so on.

The proper choice of $\alpha(s, d)$ and $\beta(s, d)$, both constrained to (1) and (2), defines the type of protection scheme adopted. For instance, $\alpha(s, d)=1$ and $\beta(s, d)=0$, as in Fig. 2b, represents DPP; $0<\alpha(s, d)<1$ and $0<\beta(s, d)$, as in Fig. 2c, produces DPP+BSR; $0<\alpha(s, d)<1$ and $\beta(s, d)=0$, as in Fig. 3a, results in PDPP; and PDPP+BSR implies $0<\alpha(s, d)<1$ and $0<\beta(s, d)$, but necessarily with $\beta(s, d) \geq[1-\alpha(s, d)(g(s, d)-1)] / g(s, d)$ for a given $\alpha(s, d)$ and with $\alpha(s, d) \geq[1-\beta(s, d) g(s, d)] /[g(s, d)-1]$ for a given $\beta(s, d)$ as in Fig. $3 \mathrm{~b}$ for $\alpha(s, d)=0.2$ and $\beta(s, d)=0.2$, respectively.

A prevailing objective in the design of EONs using static traffic is to minimize the use of the network spectrum. Therefore, with the protection schemes described above it is expected that $\alpha(s, d)$ is made as low as possible while complying with the network connectivity and admitted squeezing factor $\beta(s, d)$ negotiated in the SLA. At the same time, $\beta(s, d)$ shall be made as small as possible so that traffic contraction be mitigated under link failures.

It follows from (1) that the traffic rate carried by each one of the $g(s, d)$ participating disjoint paths under regular operation, given by $[1+\alpha(s, d)] \Lambda^{s d} / g(s, d)$, is at most equal to $[\alpha(s, d)+\beta(s, d)] \Lambda^{s d}$ which is then the bandwidth commitment level at which compliance with the SLA is tight and spectrum usage is as parsimonious as possible, thus reaching the best possible compromise between the conflicting needs to save spectrum and to comply with the terms of the SLA. It is clear in (2) that a reduction in $\beta(s, d)$ requires an increase in $\alpha(s, d)$ and vice-versa. Therefore, there is a strict trade-off between total network bandwidth reduction and admitted bandwidth squeezing under link failures.

Figs. 3a and $3 \mathrm{~b}$ highlight such compromise between $\alpha(s, d)$ and $\beta(s, d)$. While the PDPP solution of Fig. 3a does not permit squeezing (by defining $\beta(s, d)=0$ ), which leads to $\alpha(s, d)=0.5$ for $g(s, d)=3$, the alternative PDPP+BSR of Fig. 3b, by allowing squeezing with $\beta(s, d)=0.2$, achieve a reduction of $\alpha(s, d)$ from 0.5 to 0.2 in (2), thus reducing the usage of the network spectral resources. Notice also that in multi-path routing, differential delay is an issue that must be tackled for the proper functioning of the network. However, the authors in [20] and [21] address this problem and enable its practical operation. 


\section{Multiple Path Failures}

The model presented in $[\mathrm{III}-\mathrm{C}$ and the given discussions are restricted to single link failures. The model present in this section extends the single link failure scenario so that it can work against any given number of failing paths for a pair $s, d$ insofar as enough redundant connectivity options are provided by the network. If $N_{F}(s, d)$ means the number of failing paths among the $g(s, d)$ participating paths for work and protection of pair $s, d$, then $2 \leq N_{F}(s, d)<g(s, d)$ refers to a multiple failure scenario.

In order to provide this guarantee when $N_{F}(s, d)$ of the $g(s, d)$ participating link-disjoint paths fail, it is necessary that the network may then provide a total bandwidth of at least $[1-\beta(s, d)] \Lambda^{s d}$ required by the SLA. This provision requires that $[1+\alpha(s, d)] \Lambda^{s d}-N_{F}(s, d) \cdot X\left(N_{F}\right) \geq$ $[1-\beta(s, d)] \Lambda^{s d}$, where $X\left(N_{F}\right)$ is the maximal bandwidth to be reserved for work or protection in each of the $g(s, d)$ participating link-disjoint paths:

$$
X\left(N_{F}\right) \leq\left\{[\alpha(s, d)+\beta(s, d)] / N_{F}(s, d)\right\} \cdot \Lambda^{s d},
$$

with equality holding for maximal allowed squeezing. Moreover, the system must also comply with the following condition for normal operation with no squeezing:

$$
g(s, d) X\left(N_{F}\right) \leq[1+\alpha(s, d)] . \Lambda^{s d} .
$$

From (3) and (4), it follows that:

$$
X\left(N_{F}\right) \leq \min \left\{\frac{[\alpha(s, d)+\beta(s, d)]}{N_{F}(s, d)}, \frac{1+\alpha(s, d)}{g(s, d)}\right\} . \Lambda^{s d} .
$$

Moreover, operation with $N_{F}(s, d)<g(s, d)$ failing paths requires that:

$$
\left[g(s, d)-N_{F}(s, d)\right] X\left(N_{F}\right) \geq[1-\beta(s, d)] . \Lambda^{s d},
$$

so that:

$$
X\left(N_{F}\right) \geq \frac{[1-\beta(s, d)]}{\left[g(s, d)-N_{F}(s, d)\right]} \cdot \Lambda^{s d} .
$$

Normal operation also requires that $g(s, d) \cdot X\left(N_{F}\right) \geq \Lambda^{s d}$, so that:

$$
X\left(N_{F}\right) \geq \frac{\Lambda^{s d}}{g(s, d)} .
$$

From (5) and (6), it follows that

$$
X\left(N_{F}\right) \geq \max \left\{\frac{[1-\beta(s, d)]}{g(s, d)-N_{F}(s, d)}, \frac{1}{g(s, d)}\right\} \cdot \Lambda^{s d} .
$$

From (5) and (9), it follows that a solution for $X\left(N_{F}\right)$ will exist if and only if:

$$
\begin{array}{r}
\max \left\{\frac{[1-\beta(s, d)]}{g(s, d)-N_{F}(s, d)}, \frac{1}{g(s, d)}\right\} \\
\leq \min \left\{\frac{[\alpha(s, d)+\beta(s, d)]}{N_{F}(s, d)}, \frac{1+\alpha(s, d)}{g(s, d)}\right\} .
\end{array}
$$

\section{E. Feasibility regions on the $(\alpha, \beta)$-plane}

From inequality (10), feasibility regions are now derived on the $(\alpha, \beta)$-plane. For this purpose, since all parameters in (10) are function of the same source-destination pair $(s, d)$, the latter is hereby omitted for the sake of brevity. There is a compromise between the protection overhead parameter $\alpha$ and the bandwidth squeezing factor $\beta$. This compromise is bound by Eq. (10), which establishes a feasibility condition on the $(\alpha, \beta)$-plane as a function of the network connectivity parameter $g$ and the tolerable risk parameter $N_{F}$. Since $g$ and $N_{F}<g$ are both small integers, let us then analyse the feasibility condition on the $(\alpha, \beta)$ pair for some noteworthy $\left(g, N_{F}\right)$ pairs.

Normal operation will split the transmission among $g$ linkdisjoint paths, of which any $N_{F}$ may fail, thus generating the loss of a fraction $\beta$ of the throughput. Then, the left-hand side of (10) is given by:

$$
\max \left\{\frac{1-\beta}{g-N_{F}}, \frac{1}{g}\right\}=\left\{\begin{array}{rl}
\left(\frac{1-\beta}{g-N_{F}}\right) & \text { if } \quad \beta<N_{F} / g \\
\left(\frac{1}{g}\right) & \text { if } \quad \beta>N_{F} / g
\end{array},\right.
$$

and the right-hand side is:

$\min \left\{\frac{\alpha+\beta}{N_{F}}, \frac{1+\alpha}{g}\right\}=\left\{\begin{array}{cc}\frac{\alpha+\beta}{N_{F}}, \text { if } & \left(g-N_{F}\right) \cdot \alpha+g \cdot \beta<N_{F} \\ \frac{1+\alpha}{g}, \text { if } & \left(g-N_{F}\right) \cdot \alpha+g \cdot \beta>N_{F}\end{array}\right.$.

Figs. 4 and 5 show equations (11) and (12) respectively written on the $[\alpha \geq 0,0 \leq \beta \leq 1]$ strip of the $(\alpha, \beta)$-plane where each of them is valid. Accordingly, one may write:

- On the lower triangular region of Fig. 5, hereby called region 1, given by $\left(g-N_{F}\right) \cdot \alpha+g \cdot \beta<N_{F}$, it is required by (10) that $\frac{1-\beta}{g-N_{F}} \leq \frac{\alpha+\beta}{N_{F}}$, so that $\left(g-N_{F}\right) \cdot \alpha+g \cdot \beta>$ $N_{F}$. However, this condition is not met in any point of region 1 , which is defined by $\left(g-N_{F}\right) \cdot \alpha+g \cdot \beta<N_{F}$. Therefore, $(\alpha, \beta)$ is infeasible on region 1 .

- On region 2 , defined by $\frac{N_{F}-\left(g-N_{F}\right) \cdot \alpha}{g}<\beta<\frac{N_{F}}{g}$ it is required by (10) that $\frac{1-\beta}{g-N_{F}} \leq \frac{1+\alpha}{g}$ so that $\left(g-N_{F}\right) . \alpha+$ $g . \beta \geq N_{F}$, a condition that is met on all points of region 2. Therefore, $(\alpha, \beta)$ is feasible on region 2 .

- On region 3, where $\beta>\frac{N_{F}}{g}$, it is required by (10) that $\frac{1}{g} \leq \frac{1+\alpha}{g}$, which is valid everywhere, so $(\alpha, \beta)$ is feasible on region 3.

In summary, $(\alpha, \beta)$ is infeasible if $\left(g-N_{F}\right) \cdot \alpha+g \cdot \beta<N_{F}$ and feasible otherwise.

Fig. 6 displays the two boundaries (of Figs. 4 and 5) on the same space, showing that they divide it into the three regions defined above, where inequality (10) must be applied to make squeezed protection feasible.

It is apparent from Fig. 6 that $\beta$ may reach zero (i.e. no squeezing needed) if $\alpha>\frac{N_{F}}{g-N_{F}}$. However, $\frac{N_{F}}{g-N_{F}}>1$ if $N_{F}>\frac{g}{2}$, thus requiring that $\alpha>1$ to avoid squeezing if more than a half of the participating paths fail. In theory, setting $\alpha>1$ is not a problem. In practice, though, the motivation for squeezing comes from the need to be more parsimonious in 


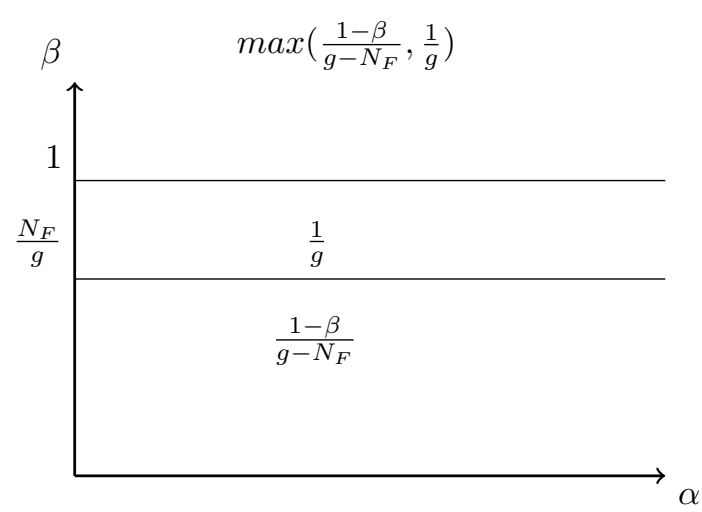

Fig. 4: Representing of left side of (10) on the $(\alpha, \beta)$-plane

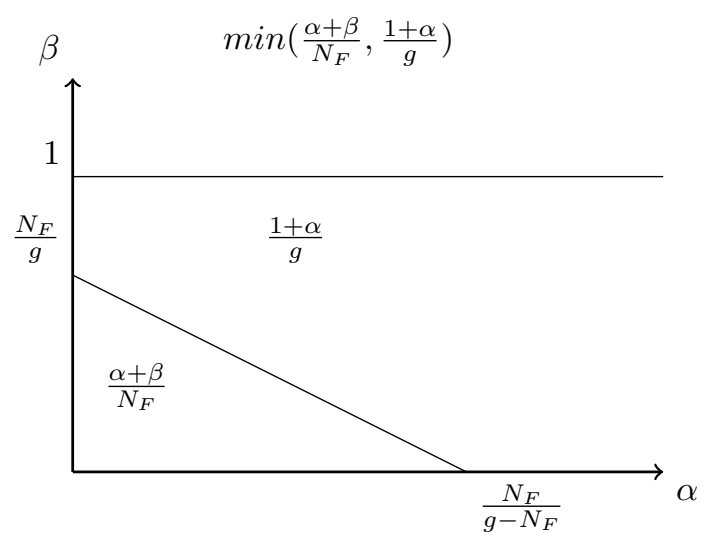

Fig. 5: Representing of right side of (10) on the $(\alpha, \beta)$-plane

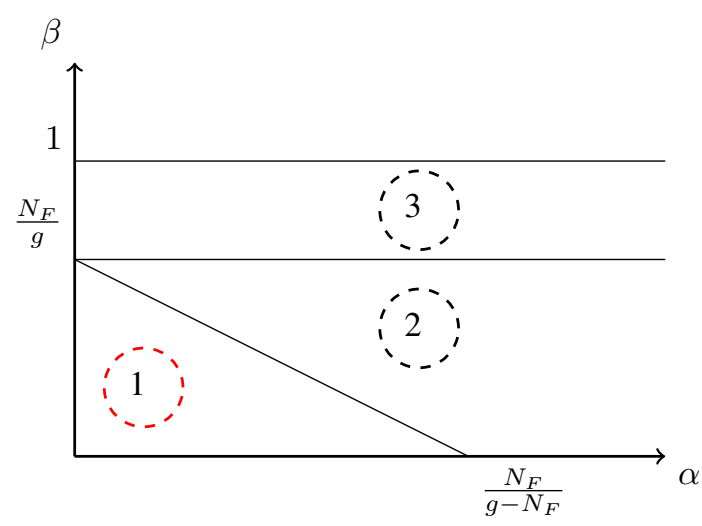

Fig. 6: Feasibility regions on the $(\alpha, \beta)$-plane. Shaded region 1 is infeasible. The others are feasible.

the use of spectral resources than dedicated protection (DP), where $\alpha=1$. Then, if one wishes to restrain $\alpha$ from exceeding 1 , it is necessary to truncate Fig. 6 at $\alpha=1$, as suggested in Fig. 7 , in order to account for the additional constraint given by $\alpha \leq 1$. Then, the squeezing factor $\beta$ cannot be taken below $2 N_{F} / g-1=1 / 3$ for $\left(g, N_{F}\right)=(3,2)$ at $\alpha=1$. The 3 straight lines depicted on Fig. 7 are the upper bounds of the infeasible regions generated by $\left(g, N_{F}\right)=(3,2)$ in red, $\left(g, N_{F}\right)=(2,1)$ in black, and $\left(g, N_{F}\right)=(3,1)$ in blue, where the number $g$ of disjoint paths between source and destination is given by

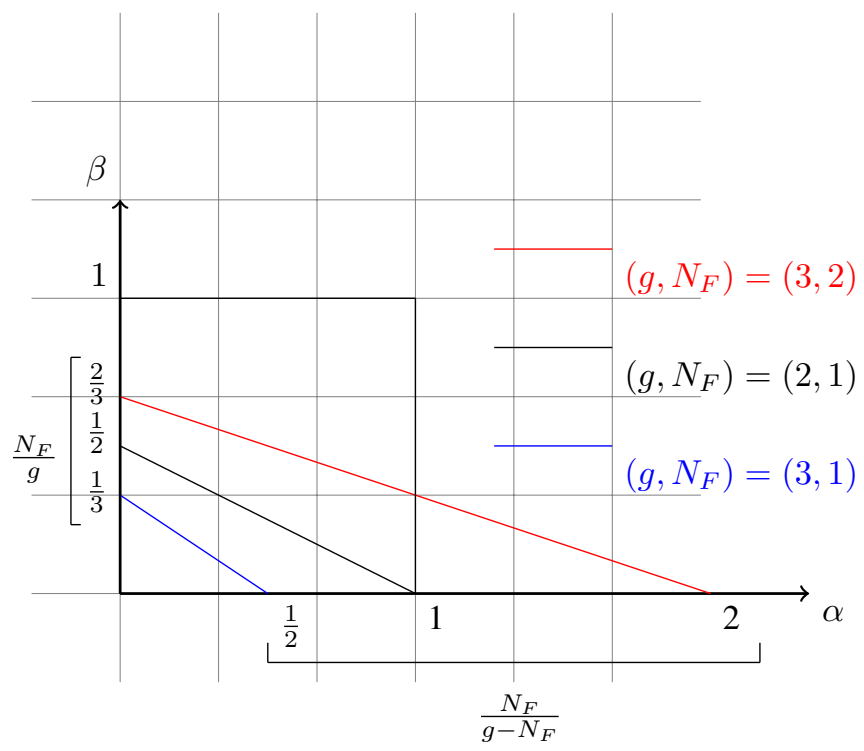

Fig. 7: Feasibility regions on the $(\alpha, \beta)$-plane for $\left(g, N_{F}\right)=(2,1)$, $(3,1)$ and $(3,2)$.

the physical topology, and the number $N_{F}$ of failures to be tolerated is derived from the risk model and the SLA. The black straight line for $\left(g, N_{F}\right)=(2,1)$ refers to the standard case of DPP, in which only a single failure is tolerated against a single backup path. In the case of the blue straight line for which $\left(g, N_{F}\right)=(3,1)$, a second backup path is added while keeping the single failure allowance, resulting in a relaxation of the feasibility compromise. In the case of the red straight line for which $\left(g, N_{F}\right)=(3,2)$, the same two backup paths are required to protect against two failing paths, thus severely hardening the feasibility compromise and forcing the squeezing factor to be at least $1 / 3$ if the bandwidth overhead factor $\alpha$ is kept below $100 \%$.

In principle, both $\alpha$ and $\beta$ should be made as small as possible in order to minimize spectrum usage and throughput loss, respectively. Fig. 7 illustrates the compromise between the minimization of $\alpha$ and $\beta$ as a function of the number $g$ of participating link-disjoint paths, which is limited by the physical topology; and the number of path failures $N_{F}$ to be tolerated while still guaranteeing that squeezing will not exceed the squeezing factor $\beta$ agreed upon in the SLA.

The drive to minimize both $\alpha$ and $\beta$ leads to their placement on the straight line that separates the feasible and infeasible regions in the $(\alpha, \beta)$-plane, shown on Fig. 7 for three cases:

- $\left(g, N_{F}\right)=(2,1)$, represented in black, in which the point $(\alpha, \beta)=(1,0)$ represents the legacy benchmark of dedicated protection;

- $\left(g, N_{F}\right)=(3,1)$, represented in blue, in which a third participating path $(g=3)$ leads to a relaxation of the compromise between $\alpha$ and $\beta$ allowing $\beta=0$ for $\alpha \geq 1 / 2$ and $\beta=1 / 3$ for $\alpha=0$;

- $\left(g, N_{F}\right)=(3,2)$, represented in red, in which the need to tolerate two failing paths $\left(N_{F}=2\right)$ among the three participants $(g=3)$ makes the compromise between $\alpha$ and $\beta$ much more restrictive, forcing the loss of $1 / 3$ of the throughput under $\alpha=1$ and $2 / 3$ under $\alpha=0$. In theory, 
$\alpha$ would have to be 2 to avoid any loss of throughput $(\beta=0)$.

\section{F. Nodal Requirements}

EONs emerged with the advent of technologies as bandwidthvariable transponders (BV-Ts) and bandwidth-variable optical switches (BV-OXCs), also referred to as reconfigurable optical add-drop multiplexer (ROADM).

BV-Ts enable a trade-off between spectral efficiency and transmission reach, using spectrally efficient modulation formats (e.g., PM-8PSK, PM- 16QAM, PM-64QAM) for shortreach connections, and more robust but less efficient modulation schemes (e.g. PM-QPSK, PM-BPSK) for long-haul links [22]. Therefore, BV-Ts are able to trade spectral efficiency off against transmission reach [1].

The main functions of a BV-OXCs are to forward a spectrum slice (transparently, i.e. entirely in the optical domain) from an input fiber to an output fiber, as well as provide add and drop lines where BV-Ts are plugged to the BV-OXC so that the signal from the upper layer can be inserted into the optical network (add) and removed from it (drop). When grooming is employed, BV-Ts are also used at BV-OXCs present at intermedia points in the route of an end-to-end traffic in order to pass the signal from the optical to electrical domain $(\mathrm{O} / \mathrm{E}$ conversion), groom it with traffic from different end points and send the combined traffic back to the optical domain (E/O conversion). Due to traffic elasticity in EONs, BV-OXCs need to configure its switching window in a flexible manner according to the spectral width of the incoming optical signal [1]. This functionality is typically performed by the use of Bandwidthvariable spectrum selective switches (BV-SSSs), which can cross-connect optical signals with different flexible bandwidth. In [1, 22] different BV-OXC architectures are explained in details.

Turning now to the survivability requirements discussed in the last previous sections, it is required that nodes be enabled to fully exploit the connectivity of the network physical topology, so that the $g$ disjoint paths may be active to support the connection and protect it against their failures. This means that the nodes may in general need to have more transponders than in classical schemes which use $g=2$. However, as $g$ is taken to higher values than 2 , the transponder bit rates decrease in the same proportion, so their unit cost decreases too. In [23], the authors show a table of available transponder profiles in which transponder costs are reduced by a factor of 3 when bit rates are reduced by a factor of 4 . These numbers suggest only a modest increase in CAPEX cost of the nodes with the proposed scheme. Some OPEX growth is also expected due to the additional footprint and energy consumption. On the other hand, the additional revenue brought by the ability to provide service to more users should more than offset the additional costs. In order to allow flexibility for service differentiation in the assignment of spectrum-prodigal protection features, sliceable bandwidth-variable transponders have been presented [24] to share its entire capacity among multiple flows. In a sliceable BV-T, multiple independent data flows can be simultaneously generated and transmitted to different destination. Therefore, optical flows can be aggregated or sliced based on traffic needs such as destination as well as security and protection overheads they agree to pay for.

In the next Section, the proposed MILP formulation and some analysis are presented, in which $\beta(s, d)$ is specified for each $(s, d)$ pair according with a given SLA, $\alpha(s, d)$ and $g(s, d)$.

\section{MILP FORMULATION}

\section{A. Notation}

- $s$ and $d$ denote the source and destination nodes of the traffic demands in the network, respectively.

- $i$ and $j$ denote originating and terminating nodes of a variable bandwidth lightpath, respectively.

- $m$ and $n$ denote endpoints of a physical link in the network.

- $z$ denotes the kind of modulation format from a set of $M$ available modulation formats.

\section{B. Given}

- $G=(N, E)$ : A graph where $N$ is the set of nodes and $E$ is the set of edges (i.e. fiber links) connecting the nodes.

- $\Lambda^{s d}$ : Traffic matrix element, it corresponds to the node pair $s-d$ whose value is the average traffic flow from node $s$ to node $d$ (in Gbit/s).

- Protection overhead $\alpha(s, d)$ : it is the fractional excess bandwidth reserved for protection of demand $\Lambda^{s d}$, so that the total bandwidth reserved for working and protection is $[1+\alpha(s, d)] \Lambda^{s d}$.

- Maximum squeezed bandwidth ratio: $\beta(s, d)$, where [1$\beta(s, d)]$ is the minimum admitted bandwidth fraction after a link failure, as agreed in the SLA on pair $s-d$.

- Physical degree $g(s, d)$ : it is the number of link-disjoint paths that carry traffic flow from node $s$ to node $d$. Notice that $g(s, d)$ is limited by the physical degrees of nodes $s$ and $d$ and the physical topology of the network.

- $N_{F}(s, d)$ : it is the number of failing paths among the $g(s, d)$ link-disjoint paths.

- F: Filter Guard Band (FGB), which represents the minimum spectrum width between wavebands. In this paper, we assume $F$ as the amount of frequency slots.

- slot width: $\Omega$.

- $d_{m n}$ : Distance between the nodes $m$ and $n$ on physical topology.

- Spectral efficiency of the modulation format $z: \eta_{z}$, where $z \in 1, \ldots, M$.

- Maximum reach for a lightpath using modulation format $z: d_{z}$, where $z \in 1, \ldots, M$.

- $\chi$ : An arbitrarily large number (greater than the number of slots available) which allows formation of constraints from integer slot allocation variables.

\section{Variables}

- Lightpath bandwidth $V_{i j}$ : bandwidth of an elastic lightpath which originates from node $i$ and terminates at node $j$ in the virtual topology (in Gbit/s). 
- Lightpath indicator $b_{i j}$ : The value of the lightpath indicator is 1 if there is a lightpath from node $i$ to node $j$. Otherwise, the value is 0 .

- $\lambda_{i j}^{s d}$ : It is the component of traffic due to a node pair $s$-d offered onto a lighpath from node $i$ to node $j$ (in Gbit/s).

- $B_{i j}^{s d}$ : A binary variable to indicate whether a fraction of traffic from node $s$ to node $d$ is routed through a lightpath from node $i$ to node $j$. $B_{i j}^{s d}$ equals to 1 if $\lambda_{i j}^{s d}>0$; equals to 0 if $\lambda_{i j}^{s d}=0$.

- $p_{i j}$ : The number of slots of an elastic lightpath from node $i$ to node $j$ in the physical topology (integer variable).

- $P_{m n}^{i j}$ : The number of slots that a lightpath from node $i$ to node $j$ uses in a fiber link $m$ - $n$ (integer variable).

- $A_{m n}^{i j}$ : A binary variable to indicate whether the lightpath from node $i$ to node $j$ passes through a link $m-n$. $A_{m n}^{i j}$ equals to 1 if $P_{m n}^{i j}>0$; equals to 0 if $P_{m n}^{i j}=0$.

- Modulation format indicator $e_{i j}^{z}$ : A binary variable that indicates if an elastic lightpath from node $i$ to node $j$ employs the modulation format $z$.

- $S_{i j}$ : Integer variable that denotes the starting frequency for lightpath $i-j$.

- $W_{i j, k t}$ : A binary variable that equals 1 if the starting frequency of lightpath $i-j$ is smaller than the starting frequency of lightpath $k$-t. (i.e, $S_{i j}<S_{k t}$ ) and 0 otherwise.

- $C$ : Number of assigned spectrum slots. The aim of the solutions presented in this paper is to minimize the number of assigned spectrum slots $(C)$ that is required to attend a set of demands with agreed protection and squeezing capabilities.

\section{Proposed MILP formulations}

The formulations proposed in this paper are based on the concepts described in Section II, whereas different strategies of lightpath protection are used and the optimum virtual topology design (VTD) and RMSA solution under grooming and different modulation formats is found.

$$
\text { Minimize : } C
$$

\section{- subject to:}

\section{Grooming and SLA Constraints:}

$$
\sum_{j} \lambda_{i j}^{s d}-\sum_{j} \lambda_{j i}^{s d}=\left\{\begin{array}{rrr}
([1+\alpha(s, d)]) \cdot \Lambda^{s d} & \text { if } \quad i=s \\
-([1+\alpha(s, d)]) \Lambda^{s d} & \text { if } \quad i=d \\
0 & \text { if } \quad i \neq s, d
\end{array}\right.
$$

$$
\lambda_{i j}^{s d} \leq\left\{[\alpha(s, d)+\beta(s, d)] / N_{F}(s, d)\right\} \Lambda^{s d} \quad \forall s, d, i, j
$$

$$
\lambda_{i j}^{s d} \leq\{[1+\alpha(s, d)] / g(s, d)\} \Lambda^{s d} \quad \forall s, d, i, j
$$

$$
\lambda_{i j}^{s d} \geq \frac{[1-\beta(s, d)]}{\left[g(s, d)-N_{F}(s, d)\right]} \Lambda^{s d}-\chi \cdot\left(1-B_{i j}^{s d}\right),
$$

$$
\begin{gathered}
\lambda_{i j}^{s d} \geq \frac{\Lambda^{s d}}{g(s, d)}-\chi\left(1-B_{i j}^{s d}\right), \\
B_{i j}^{s d} \geq \lambda_{i j}^{s d} / \chi \quad \forall s, d, i, j \\
\sum_{s d} \lambda_{i j}^{s d}=V_{i j} \quad \forall i j \\
V_{i j} / \chi \leq b_{i j} \quad \forall i j
\end{gathered}
$$

Lightpath bandwidth setting, modulation format assignment and distance requirement:

$$
\begin{gathered}
p_{i j} \geq\left(\frac{V_{i j}}{\Omega \eta_{z}}\right)-\left(1-e_{i j}^{z}\right) \chi \quad \text { for } z=1,2, \ldots, M \\
p_{i j} \leq\left(\frac{V_{i j}}{\Omega \eta_{z}}\right)+1+\left(1-e_{i j}^{z}\right) \chi \quad \text { for } z=1,2, \ldots, M \\
p_{i j} \leq \chi \sum_{z} e_{i j}^{z} \\
\sum_{z} e_{i j}^{z} \leq 1 \\
\sum_{z} e_{i j}^{z} \leq \chi V_{i j} \\
\sum_{z} e_{i j}^{z} \geq \frac{V_{i j}}{\chi} \\
\sum_{m n} A_{m n}^{i j} d_{m n} \leq \sum_{z} d_{z} e_{i j}^{z}
\end{gathered}
$$

Slot flow conservation and link usage indicator:

$$
\begin{gathered}
\sum_{n} P_{m n}^{i j}-\sum_{n} P_{n m}^{i j}=\left\{\begin{array}{r}
p_{i j} \quad m=i \\
-p_{i j} m=j \\
0 \quad m \neq i, j \\
\sum_{i j}\left(P_{m n}^{i j}+F A_{m n}^{i j}\right)-F \leq C \quad \forall m n \\
A_{m n}^{i j} \geq \frac{P_{m n}^{i j}}{\chi} \quad \forall i j, m n
\end{array} \quad \forall i j, m\right. \\
A_{m n}^{i j}+A_{m l}^{i j} \leq 1 \quad \forall i, j, m ; \quad n \neq l
\end{gathered}
$$

Link-Disjoint Paths Constraint:

$$
\sum_{i j} B_{i j}^{s d} A_{m n}^{i j} \leq 1 \quad \forall s, d, m, n
$$

Spectrum Continuity and Consecutive Slots Constraints:

$$
\begin{gathered}
p_{i j}+S_{i j} \leq C \quad \forall i j \\
S_{i j} \geq 0, p_{i j} \geq 0 \quad \forall i j \\
W_{i j, k t}+W_{k t, i j} \leq 1 \quad \forall i j, k t: i j \neq k t
\end{gathered}
$$




$$
\begin{array}{r}
W_{i j, k t}+W_{k t, i j} \geq\left[\left(A_{m n}^{i j}+A_{m n}^{k t}\right)-1\right] \\
\forall i j, k t: i j \neq k t \\
p_{i j}+S_{i j}+F \leq S_{k t}+\chi\left[1-W_{i j, k t}\right] \\
\forall i j, k t: i j \neq k t \\
p_{k t}+S_{k t}+F \leq S_{i j}+\chi\left[1-W_{k t, i j}\right] \\
\forall i j, k t: i j \neq k t
\end{array}
$$

Equation (13) is the objective function, it works to minimize the highest slot number used by any physical link. Equation (14) is the traditional conservation constraints of flows [25]. It allows traffic grooming on the virtual topology. Equations 15 - 18 allow partitioning and BSR constraints [14]. The virtual hop indicator used by the source-destination node traffic is captured by Equation (19). Equation (20) expresses the fact that the traffic flow offered onto an elastic lightpath is the sum of the traffic flows offered onto the lightpath by all source-destination pairs. The existence of a elastic lightpath between nodes $i$ and $j$ is captured by Equation (21). Equations (22) and (23) are used to ensure that the number of slots for the lightpath depends on the modulation format. A lightpath is assigned to a modulation format that satisfies the constraints from (24) until (28), which implies that a single modulation format is employed to a lightpath, and its distance limitation must be satisfied. Conservation of the flow of traffic from virtual node $i$ to virtual node $j$ as it passes through the physical links is expressed in frequency slots by Equation (29). Equation (30) denotes that, in any link of the network, the utilized bandwidth (including guard-band) should not exceed the spectrum capacity of the fiber. Equation (31) is used to evaluate the guard-band overhead and Equation (32) guarantees that the traffic of a lightpath can not be partitioned in the physical topology. Equation (33) denotes that multiple lightpaths used to route the traffic from a source-destination node pair must use different physical links in order to enable the proposed traffic partitioning mechanism.

The constraint (34), along with the objective function (13), constraints (29) and (30), is used to minimize the maximum number of spectrum slots used among all demands. Equation 35 is trivial, since a connection starting frequency and its number of slots must both be positive. Equations from (36) until (39) are the traditional spectrum continuity and slot contiguity constraints, and they are similar to the constraints presented with a detailed explanation in [14].

It can be seen that the constraint (33) is non-linear. However, it is formed by the multiplication of two binary variables. Therefore, variable $w$ can be defined as $B_{i j}^{s d} \cdot A_{m n}^{i j}=Z_{s d m n}^{i j}, \sqrt{33}$ may be replaced by equations (40) to 444 that follow, and thus convert the MILP formulation into a linear problem.

$$
\sum_{i j} Z_{s d m n}^{i j} \leq 1 \quad \forall s, d, m, n
$$

$$
\begin{gathered}
Z_{s d m n}^{i j} \leq B_{i j}^{s d} \quad \forall s, d, m, n, i \\
Z_{s d m n}^{i j} \leq A_{m n}^{i j} \quad \forall s, d, m, n, i, j \\
Z_{s d m n}^{i j} \geq 0 \quad \forall s, d, m, n, i, j \\
Z_{s d m n}^{i j} \geq B_{i j}^{s d}+A_{m n}^{i j}-1 \quad \forall s, d, m, n, i, j
\end{gathered}
$$

\section{Two-STEP MILP (HEURISTIC)}

It must be noted that the problem of virtual topology design of EON over a physical substrate is known to be non-deterministic polynomial time (NP), [26]. Because of the complexity of the problem, the presented formulation model is not scalable for large networks and may be very time-consuming. So, efficient heuristics are needed.

Therefore, we claim that the complexity of the complete MILP formulation may be reduced by decomposing the problem into two steps:

- Step 1: find the virtual links ( $b_{i j}$ 's) by a two-step MILP approach, as will be illustrated in the next Section;

- Step 2: given the virtual links $b_{i j}$ 's (It means they become parameters), run a reduced MILP formulation and find $C$.

During step 2, it is assumed that the virtual topology is known beforehand. The source-destination pairs between which a certain number of virtual links $b_{i j}$ 's are required to be established are taken as inputs. The virtual topology can be created from a heuristic algorithm [25], which determines the virtual links to be established and sets their corresponding $b_{i j}$ 's to 1 and they are an input parameter for the MILP formulation.

The second step receives the set of virtual links as input and works to find the most appropriate route with an associated protection criterion, a spectrally efficient modulation format and the set of contiguous and continuous slots in the physical substrate that best comply with the objective function directives.

Note that, by breaking the problem into two sub-problems in sequence and combining their solutions, we may end up not finding the optimal solution that would be provided by the fully integrated problem, but the processing time may be substantially reduced while still acquiring an acceptable solution. Obviously, a good solution will depend on the use of efficient heuristic for the step 1. Thus, a pre-determined virtual topology is assumed, with an average virtual degree similar to the nodal degree of the network using a similar strategy to the first phase of the minimum-delay logical topology design algorithm (MLDA) heuristic from [25]. This strategy is capable of satisfying the tightest delay constraints that are physically realizable. That is important for the virtual topology due to practical latency constraints. Therefore, in this paper we will assume this strategy for the heuristic.

The MILP formulation was solved using the IBM ILOG CPLEX solver [27] hosted in an Intel i7 3.6GHz machine with $32 \mathrm{~GB}$ of RAM. 


\section{NUMERICAL EXPERIMENT}

\section{A. Small Network}

To test the effectiveness of the MILP formulation proposed in the previous section, case studies consider the 6-node physical network substrate illustrated in Figure 8 The slot width is $\Omega=12.5 \mathrm{GHz}$ and the filter guard band between wavebands is one slot. Four modulation formats $(M=4)$ are considered to be used for the established lightpaths. For each modulation format, $z$, data rate-to-bandwidth ratio, $\eta_{z}$, are: $\eta_{1}=1, \eta_{2}=2, \eta_{3}=3$ and $\eta_{4}=4 \mathrm{bit} / \mathrm{s} / \mathrm{Hz}$ and their maximum reach are $d_{1}=8000 \mathrm{~km}, d_{2}=2000$ $\mathrm{km}, d_{3}=1000 \mathrm{~km}$ and $d_{4}=500 \mathrm{~km}$, respectively. The traffic demand is the same for each source-destination pair and is analysed in the range from $40 \mathrm{Gbit} / \mathrm{s}$ to $100 \mathrm{Gbit} / \mathrm{s}$. Table I] shows possible protection schemes that may be used for each source-destination pair in the following analysis. For this study we consider in total six possible protection schemes (i.e., combinations of $\alpha, \beta, g$ and $N_{F}$ ). Each scheme is represented by an index between 1 and 6 . Notice that not every scheme can be assigned to a source-destination node pair. Those schemes with $g=3$ can be assigned just to the source-destination nodes 2-5 and $5-2$, since they are the only combinations that can use three disjoint paths.

The protection schemes are combined to form four possible scenarios of analysis, as shown in Fig 9- Fig 12 Each of these combinations are referred to by the term Scenario (I, II, III or IV) henceforth. The matrices present the protection scheme choice for each source-destination node pair in the analysed scenarios. The values in the matrices indicate the index in Table $\mathrm{I}$ of the protection scheme used for each source-destination pair. Each element in a row (column) of the matrices are associated to a source (destination) node of a connection.

The four analysed scenarios are:

- Scenario I. Full Protection $(\beta(s, d)=0)$ for every $(s, d)$ pair with: $i) \alpha(s, d)=1$, and $N_{F}(s, d)=1$ when $g(s, d)=2$; ii) $\alpha(s, d)=2$ and $N_{F}(s, d)=2$ when $g(s, d)=3$.

- Scenario II. Partial protection with: $i$ ) $50 \%$ of squeezing for the $(s, d)$ pairs with $g(s, d)=2$ and $N_{F}(s, d)=1$; ii) $33.3 \%$ of squeezing for those pairs with $g(s, d)=3$ and $N_{F}(s, d)=2$.

- Scenario III. Partial protection with: i) 50\% of squeezing for the pairs $(s, d)$ with $g(s, d)=2 ; i i)$ $66.7 \%$ of squeezing for those pairs with $g(s, d)=3$ and $N_{F}(s, d)=2$.

- Scenario IV. Partial protection with: $i$ ) $50 \%$ of squeezing for the pairs $(s, d)$ with $g(s, d)=2 ; i i)$ $33.3 \%$ of squeezing for those pairs with $g(s, d)=3$ and $N_{F}(s, d)=1$.

Fig. 13 shows the number of assigned spectrum slots found by the MILP formulation to attend the four protection scenarios described in Scenarios I-IV when the traffic for each source destination node pairs increases from 40 to $100 \mathrm{Gbit} / \mathrm{s}$. For the purpose of comparison, the scenario without any kind of protection to its demanded traffic
TABLE I: Simulation parameter values for several protection schemes.

\begin{tabular}{ccccc}
\hline index & $\left(g, N_{F}\right)$ & $\alpha$ & $\beta$ & Protection scheme \\
\hline 1 & $(2,1)$ & 1 & 0 & DPP \\
2 & $(2,1)$ & 0 & 0.5 & PDPP+BSR \\
3 & $(3,1)$ & 0 & $1 / 3$ & PDPP+BSR \\
\hline 4 & $(3,2)$ & 0 & $2 / 3$ & PDPP+BSR \\
5 & $(3,2)$ & 1 & $1 / 3$ & PDPP+BSR \\
6 & $(3,2)$ & 2 & 0 & DPP \\
\hline
\end{tabular}

$(\alpha(s, d)=0, \beta(s, d)=1) \forall(s, d)$ is shown in red dashed line. As it can be seen, for the same amount of traffic for each source destination node pairs, Scenario I, which provides DPP for every node, consumes practically the double amount of resources than any other combination of protection mechanisms analysed.

When one compares Scenario II with Scenario III, it is possible to observe that, conceding to the source destination pairs $(2,5)$ and $(5,2)$, the increase in the amount of squeezed traffic from $\beta(s, d)=33 \%$ (Scenario II) to $\beta(s, d)=66 \%$ (Scenario III) incurs in reductions to the number of used slots. Likewise, similar reductions may also be achieved with Scenario IV by reducing the number of allowed concurrent path failures from $N_{F}=2$ (Scenario II) to $N_{F}=1$ (Scenario IV) while keeping the same squeezing factor. Finally, it is important to highlight that few additional slots are required when one compares the scenario without protection (dashed red line) with Scenarios II, III and IV, which provide protection for node pairs $(2,5)$ and $(5,2)$ against two link failures and $1 / 3$ of squeezing (Scenarios II), two link failures and $2 / 3$ of squeezing (Scenario III) and single link failure and $1 / 3$ of squeezing. Mainly when it is perceived that dedicated path protection (Scenario I) applied to node pairs $(2,5)$ and $(5,2)$ requires more than the double of slots of no protection scenario.

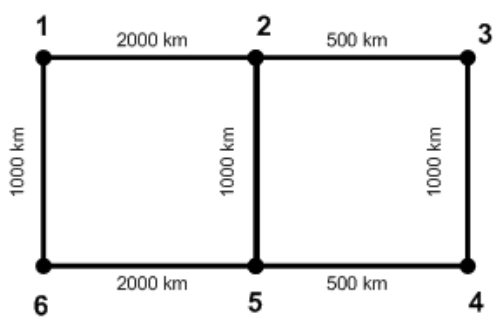

Fig. 8: Six-node network used for MILP evaluation.

TABLE II: Comparison between MILP and Two-step MILP for the number of assigned spectrum slots $(C)$, their percentage gap and CPU time for $100 \mathrm{Gbit} / \mathrm{s}$ of traffic between node pairs under protection Scenarios I, II, III and IV.

\begin{tabular}{cccc}
\hline Scenario & MILP & Two-step MILP & gap \% \\
\hline I & $40(\sim 16 \mathrm{~h})$ & $40(<2 \mathrm{~s})$ & 0 \\
\hline II & $21(\sim 16 \mathrm{~h})$ & $21(<2 \mathrm{~s})$ & 0 \\
\hline III & $20(\sim 16 \mathrm{~h})$ & $20(<2 \mathrm{~s})$ & 0 \\
\hline IV & $20(\sim 16 \mathrm{~h})$ & $20(<2 \mathrm{~s})$ & 0 \\
\hline
\end{tabular}

In Table II] we can see the CPU time to solve the MILP and two-step MILP with $100 \mathrm{Gbit} / \mathrm{s}$ of traffic between 


$$
\left(\begin{array}{llllll}
. & 1 & 1 & 1 & 1 & 1 \\
1 & . & 1 & 1 & 6 & 1 \\
1 & 1 & . & 1 & 1 & 1 \\
1 & 1 & 1 & . & 1 & 1 \\
1 & 6 & 1 & 1 & . & 1 \\
1 & 1 & 1 & 1 & 1 & .
\end{array}\right)
$$

Fig. 9: Scenario I applied for the 6-node network.

$$
\left(\begin{array}{cccccc}
. & 2 & 2 & 2 & 2 & 2 \\
2 & . & 2 & 2 & 5 & 2 \\
2 & 2 & . & 2 & 2 & 2 \\
2 & 2 & 2 & . & 2 & 2 \\
2 & 5 & 2 & 2 & . & 2 \\
2 & 2 & 2 & 2 & 2 & .
\end{array}\right)
$$

Fig. 10: Scenario II applied for the 6-node network.

$$
\left(\begin{array}{cccccc}
\cdot & 2 & 2 & 2 & 2 & 2 \\
2 & . & 2 & 2 & 4 & 2 \\
2 & 2 & . & 2 & 2 & 2 \\
2 & 2 & 2 & . & 2 & 2 \\
2 & 4 & 2 & 2 & . & 2 \\
2 & 2 & 2 & 2 & 2 & .
\end{array}\right)
$$

Fig. 11: Scenario III applied for the 6-node network.

$$
\left(\begin{array}{cccccc}
. & 2 & 2 & 2 & 2 & 2 \\
2 & . & 2 & 2 & 3 & 2 \\
2 & 2 & . & 2 & 2 & 2 \\
2 & 2 & 2 & . & 2 & 2 \\
2 & 3 & 2 & 2 & . & 2 \\
2 & 2 & 2 & 2 & 2 & .
\end{array}\right)
$$

Fig. 12: Scenario IV applied for the 6-node network.

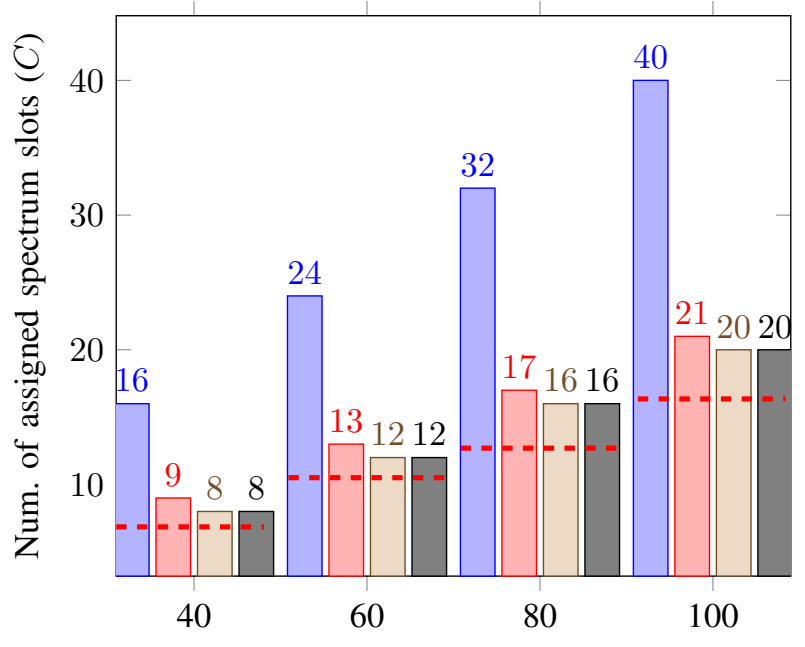

Traffic for each pair $(s, d)$ in Gbit/s

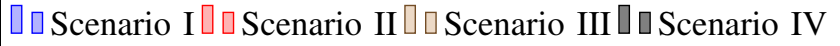

Fig. 13: Spectrum used in function of traffic.

the node pairs. The optimally gap is also shown and it is $0 \%$ for all cases, which means that the same amount of assigned spectrum slots was found. Therefore, the two- step MILP has a great performance and can solve the problem with lower computational time than MILP.

\section{B. Large Networks}

Due to the complexity of the problem for large networks, the complete strategy presented in the proposed MILP formulation is very time consuming. Indeed, to be able to search for the best route combination in the entire set of possibilities, as well as to find contiguous and continuous spectrum ranges for each route is a very complex task. Therefore, for large networks, only the two-step MILP is investigated. Three networks (VIA NETWORK, BREN and ABILENE) are analysed, where their physical substrates are presented in Fig. 17 and can be found on this website: http://www.av.it.pt/anp/on/refnet2.html All assumed parameters (slot width, filter guard band, number, spectrum efficiency and maximum reach of modulation formats) are kept identical as before.

Table III summarizes the results of the two-step MILP formulation in terms of maximum load in the virtual links $\left(\lambda_{\max }\right.$ in Gbit/s) and the number of assigned spectrum slots $(C)$ in the network for the same four protection scenarios described in Section VI-A. The generated traffic matrices employ $\Lambda^{s d}$ equal to $40 \mathrm{Gbit} / \mathrm{s}$ for all sourcedestination node pairs in the networks. Similarly to what is observed in Table II traditional DPP (Scenario I) requires much more slots from the network than any other analysed scenarios. In some cases, the resource utilization is more than the double (VIA NETWORK) or practically the double (BREN) in relation to the other scenarios. Again, this occurs because, under this kind of protection, each traffic demand needs to be assigned with the double of the required traffic when the protection scheme is resilient to one link failure and the triple for those demands committed to be resilient to two link failures (see SLA constraints). When one compares Scenario II with Scenarios III and $\mathrm{IV}$, it is possible to observe that, conceding to the source destination pairs with $g=3$, the reduction in the amount of squeezed traffic from $\beta(s, d)=66 \%$ (Scenario III) to $\beta(s, d)=33 \%$ (Scenario II) can be achieved with only small or even no increase in the number of assigned spectrum slots; and when conceding the increase in the number of allowed concurrent path failures from $N_{F}=1$ (Scenario IV) to $N_{F}=2$ (Scenario II), the same occurs. The increases in assigned slots are either small or null because few pairs of nodes, for example $(7,1)$ and $(1,7)$ on VIA NETWORK, are assigned with a protection scenario different from the protection scheme 2 (PDPP+BSR with $N_{F}=1, \alpha=0$ and $\beta=0.5$ ) in Table I and therefore the network can use idle slots in the network to transport the required additional traffic.

The results clearly show that the use of traffic partitioning along with squeezing (Scenarios II, III and IV) provides significant resource savings compared to DPP, as also observed for the previously analyzed small network. There is a clear trade off between overall required resources $(\alpha)$, degree of resilience to link failure $\left(N_{F}\right)$ and fraction of 
traffic squeezing $(\beta)$. For instance, when Scenarios II and III are compared, the same (ABILENE) or practically the same (VIA NETWORK and BREN) amount of assigned spectrum slots is required, but with a clear compromise between reducing the overall traffic routed in the network (which is related to $\alpha$ ) at the expense of providing high traffic squeezing (by setting $\alpha=0$ and $\beta=2 / 3$ in Scenario III) or relaxing the savings in the amount of traffic routed in the network on behalf of traffic squeezing mitigation (by setting $\alpha=1$ and $\beta=1 / 3$ in Scenario II. Regarding the simulation time, the final solution was found with less than 50 seconds in all scenarios. Since the amount of nodes and links in backbone optical networks are usually not very large, the simulations were to moderate networks.

Table IV summarizes the number of times each of the modulation formats, among $z_{1}, z_{2}, z_{3}$ and $z_{4}$, are used in the three analysed topologies for the two-step MILP approach with $\Lambda^{(s, d)}=40 \mathrm{Gbit} / \mathrm{s}$. The advantage of using multiple modulation formats is that the most appropriate operational scheme for EONs considers that different lightpaths can be established using different modulation formats, according to physical layer constraints [14]. It can be observed that different modulation format assignments are performed depending on the employed scenario and network topology. In addition, in all cases, the modulation format choice is spread among the four possibilities, except for the VIA NETWORK, since no link below to $500 \mathrm{Km}$ (limiting distance to $z_{4}$ ) is present, and no transparent light path above $2000 \mathrm{Km}$ (limiting distance to $z_{2}$ ) is required by the Two-Step MILP approach to minimize the number of assigned spectrum slots in either of the four analysed scenarios.

In Figs. $18(\mathrm{a}-\mathrm{c})$, the $\mathrm{x}$ axis is the ID of each fiber link for each network over study and the y axis represents the number of slots used on each fiber link for each of the analysed scenarios. One can see that, in VIA NETWORK (Fig. 18a), the number of assigned spectrum slots in the network (25 in Scenario I, 13 in Scenario II and 12 in Scenarios III and IV) coincides with the the number of used slots in several links of the network. Similar features are accompanied by BREN and ABILENE networks, but with a reduction in the fraction of links with slot usage equal to the number of assigned spectrum slots.

In order to investigate the robustness of the two-step MILP approach to traffic variations, we have also simulated cases with uniform distribution between 40,60,80 and $100 \mathrm{Gbit} / \mathrm{s}$. It means that $40,60,80$ or $100 \mathrm{Gbit} / \mathrm{s}$ are equally likely to be sent by each node to every other node. In this case, the traffic matrix is referred to as "rand" in the analysis henceforth. We observe in Fig. 18 a clear gain in terms of saved bandwidth for Scenarios II, III and IV. Fig. 19 also compares the "rand" demand to the sourcedestination pair with fixed demands $(60,80$ or $100 \mathrm{Gbit} / \mathrm{s})$. The amount of spectrum is as expected: an intermediate value between the findings for the fixed demands.

\section{Best effort squeezing with guaranteed worst case}

In the previous analysis, $\alpha(s, d)$ and $\beta(s, d)$ were provided as fixed network parameters once the required amount of squeezing, available link-disjoint paths and tolerable faults are known. While observing Figs 5, 6 and 7, note that the most efficient values of $\alpha$ and $\beta$ are in the border line of the feasible region and are conflicting values (i.e, the higher is $\beta(s, d)$, the lower is $\alpha(s, d)$ and vice-versa). Therefore, if we use the SLA required value of $\beta(s, d)$ as an upper bound, instead of a rigid value, it is possible that the MILP optimal solution may slide over the border line so that the network can provide connections with squeezing factors $\beta$ lower than their required maximal values, thus allowing for a higher amount of allocated traffic $\alpha$ in the network, but only if this can be done without compromising the reliability of other currently active connections. Then, since the network may have spare capacities, the idea is to realize such trade-off while preserving the assigned spectrum in the network $(C)$. Obviously, by adding additional traffic to the network, traffic processing is also increased. However, the network operator can now negotiate with their customers reduced squeezing factors for protection against increased traffic processing.

To solve the MILP with this approach, we can change the objective function such that it keeps minimizing $C$ while also searching for the solution with the highest squeezing reduction:

$$
\min \quad\left\{C+\theta \sum_{s d} \beta(s, d)_{v}\right\}
$$

where $\theta$ is a positive factor small enough to make $\theta \sum_{s d} \beta(s, d)_{v} \leq 1$, thus not interfering with the main aim in the objective function.

As the squeezing factor is now a variable, we define $\beta(s, d)_{v}$ as the possible values assigned to the squeezing ratio and include the following constraint to the formulation:

$$
0 \leq \beta(s, d)_{v} \leq\{\beta(s, d)\} \quad \forall s, d,
$$

where $\beta(s, d)$ is now a limiting squeezing ratio.

Let us assume Scenario II in the 6-node network, where $\beta(s, d)=0.33$ and $\alpha(s, d)=0$ for the pairs $2-5$ and 5-2 and $\beta(s, d)=0.5$ and $\alpha(s, d)=1$ for the other pairs. When $\alpha(s, d)_{v}$ and $\beta(s, d)_{v}$ are included as variables in the MILP formulation, we obtain the same value of $C$ as before and the results in Figs 14 and 15 for $\alpha(s, d)_{v}$ and $\beta(s, d)_{v}$, respectively. It is interesting to see that $C$ is sustained even though $\alpha(s, d)_{v}$ is increased for some node pairs in order to decrease $\beta(s, d)_{v}$ accordingly. Notice also from Fig. 15 that 8 of the 28 source-destinations pairs that allowed for 50\% squeezing in their SLA's did not experience any squeezing at all.

Figure 16 shows the maximum slot index in each of the network links. As it can be seen, MILP with flexible $\alpha(s, d)$ and $\beta(s, d)$ uses some slot indexes not used before, but keeping the maximum slot index. 


$$
\left(\begin{array}{cccccc}
. & 0 & 0.16 & 0 & 0 & 0 \\
0 & . & 0.41 & 1 & 1 & 0 \\
0 & 1 & . & 1 & 1 & 0 \\
0.16 & 0.41 & 1 & . & 1 & 0 \\
0 & 1 & 1 & 1 & . & 0 \\
0 & 0 & 0 & 0 & 0 & .
\end{array}\right)
$$

Fig. 14: Results of $\alpha(s, d)_{v}$ applied for the MILP formulation in the 6-node network.

$$
\left(\begin{array}{cccccc}
. & 0.5 & 0.42 & 0.5 & 0.5 & 0.5 \\
0.5 & . & 0.295 & 0 & 0.33 & 0.5 \\
0.5 & 0 & . & 0 & 0 & 0.5 \\
0.42 & 0.295 & 0 & . & 0 & 0.5 \\
0.5 & 0.33 & 0 & 0 & . & 0.5 \\
0.5 & 0.5 & 0.5 & 0.5 & 0.5 & .
\end{array}\right)
$$

Fig. 15: Results of $\beta(s, d)_{v}$ applied for the MILP formulation in the 6-node network.

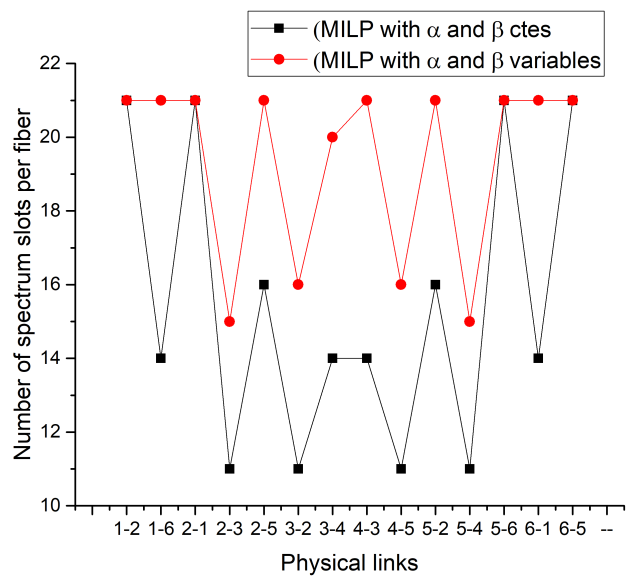

Fig. 16: Six-node network for MILP evaluation for Scenario II against $\alpha(s, d)_{v}$ and $\beta(s, d)_{v}$ with $\beta(s, d)_{v} \leq 0.33$ for s-d pairs 2-5 and 5-2; and $\beta(s, d)_{v} \leq 0.5$ for the other pairs.

\section{CONCLUding Remarks}

In this study we proposed a novel MILP formulation and a two-step MILP for different protection schemes against multiple link failures in EONs, considering the virtual topology. The proposed formulation is able to provide different protection levels, or SLA, with a BSR strategy, reducing the spectrum usage. The experiments demonstrated the effectiveness of our MILP formulation as well as our proposed two-step MILP. The present study makes several noteworthy contributions to the design of EONs with survivability, reporting advantages of PDPP and/or BSR compared to traditional dedicated protection. A natural progression of this work is to examine more closely the relation between squeezing for protection and other different protection approaches against nodes failure, SRLG etc.

The solutions discussed in this paper may enhance the requirements on the nodal architecture at the optical level. In order to balance the efficient use of spectral resources with the tolerance requirements of the users, the combination of squeezing and partitioning splits the signals among as many link-disjoint paths as possible. The resulting performance gains are thus obtained from the enhanced exploration of the network connectivity at the cost of allowing for more space diversity, which may increase the demand for optical ports and spectrumselective switches for the same amount of information, albeit at a reduced rate. The ongoing development of sliceable BVTs should provide the required functionalities, but more slices may be needed in very connected networks, thus requiring more ports and more spectrum-selective switches, if the connectivity is fully explored. More detailed techno-economic studies of architectural solutions may then be warranted in order to assess the best compromise between cost and benefit in the exploration of the network connectivity.

\section{ACKNOWLEDGMENT}

The authors would like to thank to Fapesp (Proc. 2015/24341-7), FACEPE, CAPES and CNPq for scholarships and grants and the educational support to UFBA, UFPE and UNICAMP. 
TABLE III: REAL-WORLD REFERENCE NETWORKS http://www.av.it.pt/anp/on/refnet2.html - maximum load in the virtual links ( $\left.\lambda_{\max }\right)$ and utilized spectrum slot index "C" in the Network (Two-step MILP Approach, $\Lambda^{(s, d)}=40$ Gbit/s).

\begin{tabular}{|c|c|c|c|c|c|c|c|c|c|c|c|c|}
\hline \multirow{2}{*}{ Network ID } & \multirow[t]{2}{*}{ Name } & \multirow[t]{2}{*}{$\mathrm{N}$} & \multirow[t]{2}{*}{$\mathrm{L}$} & \multirow{2}{*}{$\begin{array}{l}\text { Nodal Degree } \\
\quad(\text { mean })\end{array}$} & \multicolumn{2}{|c|}{ Scenario I } & \multicolumn{2}{|c|}{ Scenario II } & \multicolumn{2}{|c|}{ Scenario III } & \multicolumn{2}{|c|}{ Scenario IV } \\
\hline & & & & & $\lambda_{\max }$ & $C$ & $\lambda_{\max }$ & $C$ & $\lambda_{\max }$ & $C$ & $\lambda_{\max }$ & $C$ \\
\hline 1 & VIA NETWORK & 9 & $12 \times 2$ & 2.67 & 920 & $25(7.64 \mathrm{~s})$ & 473.33 & $13(7.80 \mathrm{~s})$ & 446.7 & $12(7.30 \mathrm{~s})$ & 446.7 & $12(7.30 \mathrm{~s})$ \\
\hline 2 & BREN & 10 & $11 \times 2$ & 2.20 & 1320 & $100(2.27 \mathrm{~s})$ & 666.7 & $51(2.23 \mathrm{~s})$ & 653.3 & $50(3.97 \mathrm{~s})$ & 653.3 & $50(3.86 \mathrm{~s})$ \\
\hline 3 & ABILENE & 11 & $13 \times 2$ & 2.36 & 1280 & $52(22.55 \mathrm{~s})$ & 646.7 & $26(32.17 \mathrm{~s})$ & 633.3 & $26(44.1 \mathrm{~s})$ & 633.3 & $26(43.9 s)$ \\
\hline
\end{tabular}

TABLE IV: Number of times modulation format $z=1,2,3,4$ was established under the Two-step MILP Approach applied to the three analysed large networks and four protection Scenarios. $M=4$ and $\Lambda^{(s, d)}=40 \mathrm{Gbit} / \mathrm{s}$.

\begin{tabular}{c|cccc|cccc|cccc}
\hline & \multicolumn{1}{|c|}{ VIA NETWORK } & \multicolumn{4}{c|}{ BREN } & \multicolumn{4}{c}{ ABILENE } \\
Modulation Format: & $\mathrm{z}=1$ & $\mathrm{z}=2$ & $\mathrm{z}=3$ & $\mathrm{z}=4$ & $\mathrm{z}=1$ & $\mathrm{z}=2$ & $\mathrm{z}=3$ & $\mathrm{z}=4$ & $\mathrm{z}=1$ & $\mathrm{z}=2$ & $\mathrm{z}=3$ & $\mathrm{z}=4$ \\
\hline Scenario I & 0 & 0 & 24 & 0 & 7 & 8 & 6 & 1 & 2 & 11 & 11 & 4 \\
\hline Scenario II & 0 & 0 & 24 & 0 & 6 & 8 & 6 & 2 & 2 & 12 & 13 & 1 \\
\hline Scenario III & 0 & 7 & 17 & 0 & 14 & 8 & 0 & 0 & 2 & 12 & 10 & 4 \\
\hline Scenario IV & 0 & 7 & 17 & 0 & 14 & 8 & 0 & 0 & 2 & 12 & 10 & 4 \\
\hline
\end{tabular}

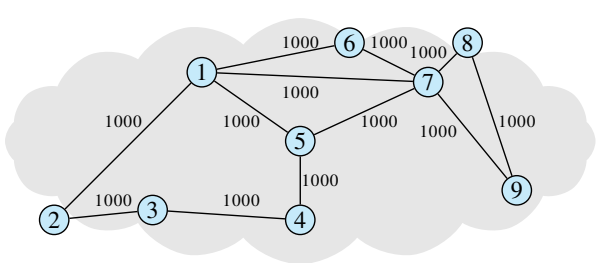

(a) VIA NETWORK.

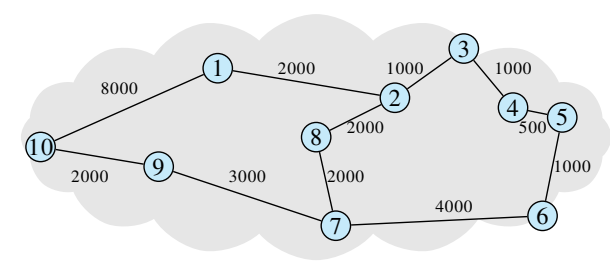

(b) BREN.

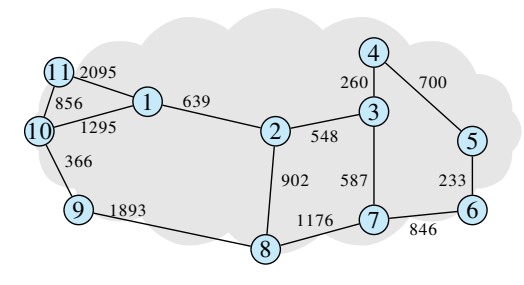

(c) ABILENE.

Fig. 17: Large networks over study (distance in $\mathrm{km}$ ).

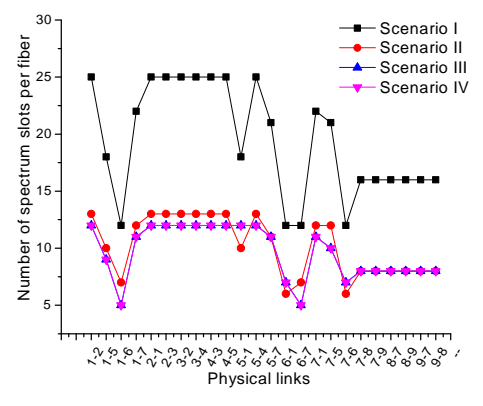

(a) VIA NETWORK

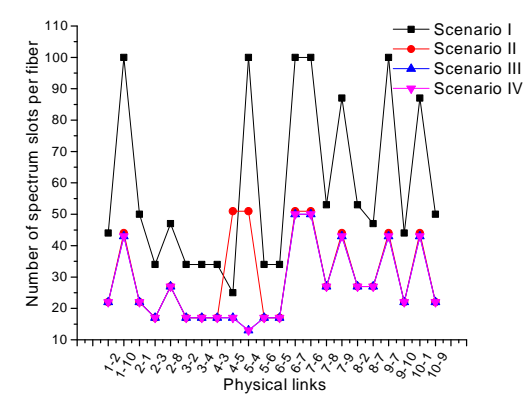

(b) BREN

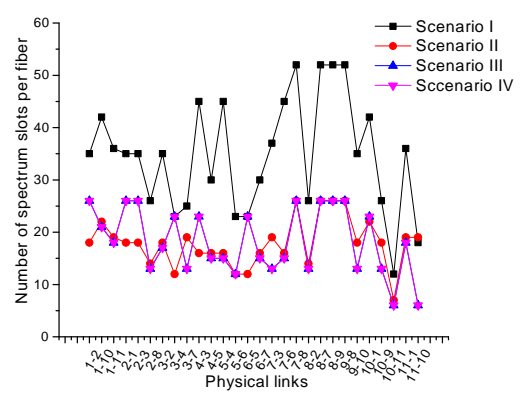

(c) ABILENE

Fig. 18: Number of used spectrum slots in each network fiber for the three large network over study under 40 Gbit/s of traffic among node pairs.

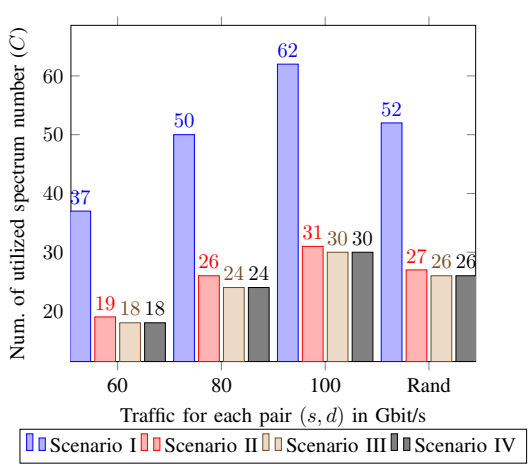

(a) VIA NETWORK

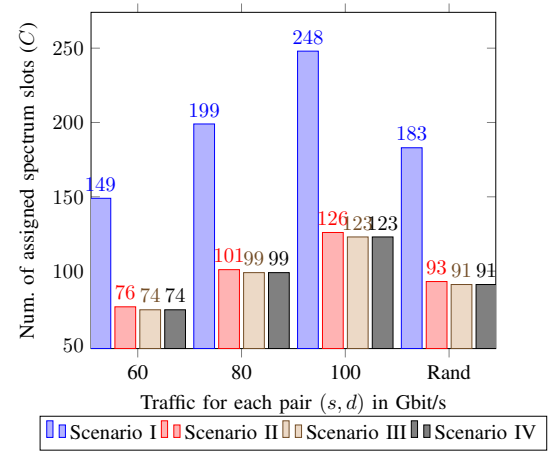

(b) BREN

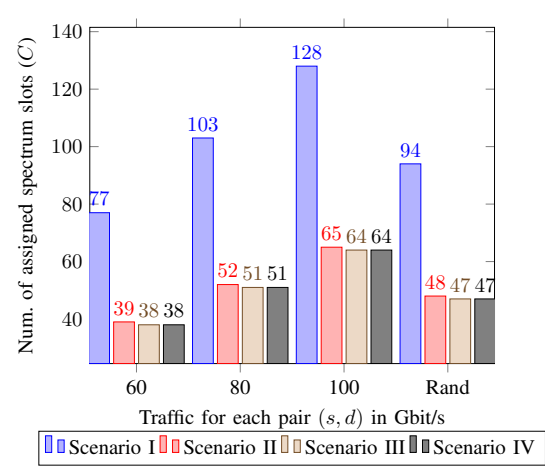

(c) ABILENE

Fig. 19: Spectrum used in function of node pair traffic. 


\section{REFERENCES}

[1] B. Chatterjee and E. Oki, Elastic Optical Networks: Fundamentals, Design, Control, and Management. CRC Press, 2020.

[2] M. Jinno, H. Takara, B. Kozicki, Y. Tsukishima, Y. Sone, and S. Matsuoka, "Spectrum-efficient and scalable elastic optical path network: architecture, benefits, and enabling technologies," IEEE Communications Magazine, vol. 47, no. 11, pp. 6673, 2009.

[3] O. Gerstel, M. Jinno, A. Lord, and S. J. B. Yoo, "Elastic optical networking: a new dawn for the optical layer?" IEEE Communications Magazine, vol. 50, no. 2, pp. s12-s20, 2012.

[4] R. T. Wong, "Telecommunications network design: Technology impacts and future directions," Networks, vol. n/a, no. n/a. [Online]. Available: https://onlinelibrary.wiley.com/doi/abs/ 10.1002/net.21997

[5] R. Girão-Silva, B. Nedic, M. Gunkel, and T. Gomes, "Shared risk link group disjointness and geodiverse routing: A trade-off between benefit and practical effort," Networks, vol. 75, no. 4, pp. 374-391, 2020. [Online]. Available: https://onlinelibrary.wiley.com/ doi/abs/10.1002/net.21931

[6] J. Rak, D. Hutchison, J. Tapolcai, R. Bruzgiene, M. Tornatore, C. Mas-Machuca, M. Furdek, and P. Smith, "Fundamentals of communication networks resilience to disasters and massive disruptions," in Guide to Disaster-Resilient Communication Networks. Springer, 2020, pp. 1-43.

[7] S. Ayoubi, Y. Zhang, and C. Assi, "A reliable embedding framework for elastic virtualized services in the cloud," IEEE Transactions on Network and Service Management, vol. 13, no. 3, pp. 489-503, 2016.

[8] G. Zhang, M. De Leenheer, and B. Mukherjee, "Optical traffic grooming in ofdm-based elastic optical networks [invited]," IEEE/OSA Journal of Optical Communications and Networking, vol. 4, no. 11, pp. B17-B25, Nov 2012.

[9] S. M. H. Ghazvini, A. G. Rahbar, and B. Alizadeh, "Load balancing, multipath routing and adaptive modulation with traffic grooming in elastic optical networks," Computer Networks, vol. 169, p. 107081, 2020.

[10] R. Ramaswami, K. Sivarajan, and G. Sasaki, Optical networks: a practical perspective. Morgan Kaufmann, 2009.

[11] Y. Sone, A. Watanabe, W. Imajuku, Y. Tsukishima, B. Kozicki, H. Takara, and M. Jinno, "Bandwidth squeezed restoration in spectrum-sliced elastic optical path networks (slice)," IEEE/OSA Journal of Optical Communications and Networking, vol. 3, no. 3, pp. 223-233, Nov 2011.

[12] L. Velasco, M. Klinkowski, M. Ruiz, and J. Comellas, "Modeling the routing and spectrum allocation problem for flexgrid optical networks," Photonic Network Communications, vol. 24, no. 3, pp. 177186, April 2012.

[13] F. Paolucci, A. Castro, F. Cugini, L. Velasco, and P. Castoldi, "Multipath restoration and bitrate squeezing in sdn-based elastic optical networks," Photonic network communications, vol. 28, no. 1, pp. 45-57, August 2014.

[14] K. Assis, R. Almeida, H. Waldman, A. Santos, M. Alencar, M. Reed, A. Hammad, and D. Simeonidou, "Sla formulation for squeezed protection in elastic optical networks considering the modulation format," IEEE/OSA Journal of Optical Communications and Networking, vol. 11, no. 5, pp. 202-212, 2019.

[15] G. Shen, H. Guo, and S. K. Bose, "Survivable elastic optical networks: Survey and perspective (invited)," Photonic Netw. Commun., vol. 31, no. 1, pp. 71-87, Feb. 2016. [Online]. Available: http://dx.doi.org/10.1007/s11107-015-0532-0

[16] K. D. R. Assis, R. C. Almeida, and H. Waldman, "Milp formulation for squeezed protection in spectrum-sliced elastic optical path networks," in IEEE International Symposium on Performance Evaluation of Computer and Telecommunication Systems (SPECTS), March 2012, pp. 1-7.

[17] R. Goscien, K. Walkowiak, M. Klinkowski, and J. Rak, "Protection in elastic optical networks," IEEE Network, vol. 29, no. 6, pp. 88-96, Nov 2015.

[18] K. D. R. Assis, A. F. Santos, R. C. Almeida, M. J. Reed, B. Jaumard, and D. Simeonidou, "Virtualization of elastic optical networks and regenerators with traffic grooming," J. Opt. Commun. Netw., vol. 12, no. 12, pp. 428-442, Dec 2020. [Online]. Available: http://jocn.osa.org/abstract.cfm? URI=jocn-12-12-428

[19] N. Shahriar, S. Taeb, S. R. Chowdhury, M. Zulfiqar, M. Tornatore, R. Boutaba, J. Mitra, and M. Hemmati, "Reliable slicing of $5 \mathrm{~g}$ transport networks with bandwidth squeezing and multi-path provisioning," IEEE Transactions on Network and Service Management, vol. 17, no. 3, pp. 1418-1431, 2020.

[20] R. Alvizu, G. Maier, M. Tornatore, and M. Pioro, "Differential delay constrained multipath routing for sdn and optical networks," Electronic Notes in Discrete Mathematics, vol. 52, pp. 277 - 284, 2016, iNOC 2015“ 7th International Network Optimization Conference. [Online]. Available: http://www.sciencedirect.com/science/ article/pii/S1571065316300427

[21] K. Yedugundla, S. Ferlin, T. Dreibholz, Özgü Alay, N. Kuhn, P. Hurtig, and A. Brunstrom, "Is multi-path transport suitable for latency sensitive traffic?" Computer Networks, vol. 105, pp. $1-21$, 2016. [Online]. Available: http://www.sciencedirect. com/science/article/pii/S1389128616301396

[22] E. Riccardi, A. Pagano, E. Hugues-Salas, G. Zervas, D. Simeonidou, A. D’Errico, M. Bohn, A. Napoli, 
D. Rafique, M. Nölle et al., "Sliceable bandwidth variable transponders for elastic optical networks: the idealist vision," in 2015 Fotonica AEIT Italian Conference on Photonics Technologies. IET, 2015, pp. 1-4.

[23] A. Eira, J. Santos, J. Pedro, and J. Pires, "Design of survivable flexible-grid $\mathrm{dwdm}$ networks with joint minimization of transponder cost and spectrum usage," in European Conference and Exhibition on Optical Communication. Optical Society of America, 2012, pp. P5-16.

[24] M. Jinno, H. Takara, Y. Sone, K. Yonenaga, and A. Hirano, "Multiflow optical transponder for efficient multilayer optical networking," IEEE Communications Magazine, vol. 50, no. 5, pp. 5665, 2012.

[25] R. Ramaswami and K. N. Sivarajan, "Design of logical topologies for wavelength-routed optical networks," IEEE J.Sel. A. Commun., vol. 14, no. 5, pp. 840-851, Jun. 1996. [Online]. Available: http://dx.doi.org/10.1109/49.510907

[26] Shahriar, Nashid, "Survivable virtual network embedding in transport networks," 2020. [Online]. Available: http://hdl.handle.net/10012/16085

[27] ILOG, Inc, "ILOG CPLEX: High-performance software for mathematical programming and optimization," 2018, see https://www.ibm.com/analytics/ cplex-optimizer. 